\title{
Translating cancer genomics into precision medicine with artificial intelligence: applications, challenges and future perspectives
}

\author{
Jia Xu ${ }^{1}$ (D) Pengwei Yang ${ }^{1} \cdot$ Shang Xue ${ }^{1} \cdot$ Bhuvan Sharma $^{1} \cdot$ Marta Sanchez-Martin $^{1} \cdot$ Fang Wang $^{1} \cdot$ Kirk A. Beaty $^{1}$. \\ Elinor Dehan ${ }^{1} \cdot$ Baiju Parikh $^{1}$
}

Received: 27 September 2018 / Accepted: 2 January 2019 / Published online: 22 January 2019

(c) The Author(s) 2019

\begin{abstract}
In the field of cancer genomics, the broad availability of genetic information offered by next-generation sequencing technologies and rapid growth in biomedical publication has led to the advent of the big-data era. Integration of artificial intelligence (AI) approaches such as machine learning, deep learning, and natural language processing (NLP) to tackle the challenges of scalability and high dimensionality of data and to transform big data into clinically actionable knowledge is expanding and becoming the foundation of precision medicine. In this paper, we review the current status and future directions of AI application in cancer genomics within the context of workflows to integrate genomic analysis for precision cancer care. The existing solutions of AI and their limitations in cancer genetic testing and diagnostics such as variant calling and interpretation are critically analyzed. Publicly available tools or algorithms for key NLP technologies in the literature mining for evidencebased clinical recommendations are reviewed and compared. In addition, the present paper highlights the challenges to AI adoption in digital healthcare with regard to data requirements, algorithmic transparency, reproducibility, and real-world assessment, and discusses the importance of preparing patients and physicians for modern digitized healthcare. We believe that AI will remain the main driver to healthcare transformation toward precision medicine, yet the unprecedented challenges posed should be addressed to ensure safety and beneficial impact to healthcare.
\end{abstract}

\section{Background}

Is artificial intelligence (AI) going to take over the world as pictured in the sci-fi movies? It has famously beaten the best-performing human beings in competitions such as Jeopardy, AlphaGo, etc., and is now crawling into our daily life without notice. Autonomous vehicles, smart homes, chat bots, individualized marketing, fraud detection, and high-frequency automated trading are some examples of AI empowering humans to live in a more efficient and personalized way. AI augments and is complement to, not a replacement for human intelligence and intuition, where its goal is to help humans become faster and smarter in certain tasks.

Healthcare, an industry that is long governed by medical professionals, is also benefitting from AI. Progress in artificial intelligence and machine learning, along with the accessibility

Jia Xu and Pengwei Yang have contributed equally.

Jia Xu

xujia@us.ibm.com

1 IBM Watson Health, Cambridge, MA, USA of cloud scaling for big data storage, and integration of health records have expanded the potential for personalized medicine (Syrjala 2018). Information can be automatically extracted and summarized from electronic medical records or from manually written doctor notes by natural language processing (NLP) (Bedi et al. 2015; Chang et al. 2016; Garvin et al. 2018; Meystre and Haug 2006; Miotto et al. 2016; Osborne et al. 2016). Through application of deep learning on medical imaging data, AI has outperformed expert pathologists and dermatologists in diagnosing metastatic breast cancer, melanoma, and several eye diseases (De Fauw et al. 2018; Ehteshami Bejnordi et al. 2017; Haenssle et al. 2018). AI also contributes to innovations in liquid biopsies and pharmacogenomics, which will revolutionize cancer screening and monitoring, and improve the prediction of adverse events and patient outcomes (Cohen et al. 2018; Low et al. 2018). Moreover, AI applications are already playing an important role in fields like gene-editing CRISPR and drug discovery (Abadi et al. 2017; Yu et al. 2017). AI-powered services, such as monitoring health status and suggesting actions to improve well-being through the use of mobile devices and the internet of things (IoT), are entering the market. 
In the field of cancer genomics, the availability of multiomics data, genotype-phenotype data through genomewide association studies (GWAS), and literature mining has fostered the development of advanced AI techniques and solutions, which allow medical professionals to deliver personalized care through precision medicine (Li et al. 2018; Szymczak et al. 2009; Telenti et al. 2018). Precision medicine is an emerging approach for disease prevention and treatment based on the specific genetics, environment, and lifestyle choices of an individual patient. AI systems are capable of identifying individual drug-response variability (Kalinin et al. 2018; Lin et al. 2018), making recommendations based on patterns learned from vast amounts of public and proprietary data sources, and can help extend the frontier of personalized medicine and specifically of cancer genomics. In this review, we will focus on the existing solutions and applications of $\mathrm{AI}$ in the field of cancer genomics and how they are shaping the precision oncology field.

\section{What is artificial intelligence}

$\mathrm{AI}$ is the combination of theories, algorithms, and computing frameworks, facilitating various tasks that require human intelligence such as reasoning, decision-making, speech recognition, language understanding, and visual perception. It is one term that encompasses numerous methods such as logic (rule-based), machine learning (ML), deep learning, NLP, and computer vision. AI can help to significantly speed up the process of analyzing vast amounts of data, leverage patterns in the data, and result in faster and better advised decision-making. Sophisticated predictive models are built using algorithms that mine the patterns from data and predict outcomes. As the availability of data in a domain increases, so does the adoption and utilization of such disruptive technologies. With the advent of Big Data and the ever-increasing storage and computing power, the challenge has shifted from collecting data to turning it into meaningful and actionable insights.

\section{How next-generation sequencing is changing the landscape of cancer genomics}

Next-generation sequencing (NGS) is being applied broadly as a valuable method for gaining insights into the genomic profile of a tumor. The ability to simultaneously sequence millions of DNA fragments in a single sample to detect a wide range of aberrations provides a comprehensive profile of the tumor. Due to comprehensive detection of aberrations, combined with improvements in reliability, sequencing chemistry, pipeline analysis, data interpretation, and cost, the adoption of NGS for clinical purposes has grown tremendously (Pennell et al. 2018). Cancer panels are designed specifically to detect clinically relevant somatic mutations with high confidence. Germline mutations in cancer-predisposing genes such as BRCA1/2 are also detected to assess cancer risk. In 2017, the FDA approved several NGS-based panels related to oncology: Oncomine Dx Target Test, Praxis Extended RAS Panel, MSK-IMPACT, and FoundationOne CDx. Recent FDA approval of NTRK gene fusions for tumoragnostic indications also expands the clinical utilization of NGS (Larotrectinib FDA approval).

Liquid biopsy holds great promise due to its noninvasive nature. Multiple studies have demonstrated the application of liquid biopsy for cancer diagnosis, prognosis, and drug-response monitoring (Palmirotta et al. 2018). Cell-free DNA (cfDNA) released by dying tumor cells, cell-derived vesicles termed exosomes, and circulating tumor cells (CTCs), which shed from the tumor and enter the vasculature system, are often used as a source for tumor DNA. Importantly, a variety of research groups have shown that NGS-sequencing protocols can be modified to achieve sensitivity levels comparable to the standard sequencing procedures (Aravanis et al. 2017), but its implementation in clinical practice is pending confirmation via clinical trials.

The Cancer Genome Atlas (TCGA) project highlights how NGS screens can facilitate the discovery of novel oncogenic mechanisms and patient stratification. The data have been used to elucidate functionally relevant oncogenic mechanisms across multiple tumor types (Cancer Genome Atlas Research Network et al. 2013; SanchezVega et al. 2018; Cava et al. 2018). In a recent study, the regulatory role of F-box/WD repeat-containing protein 7 (Fbw7) in cancer cell oxidative metabolism is discovered (Davis et al. 2018) using ML algorithms. Molecular subtypes discovered in the pan-cancer studies also help to personalize the treatment and improve patient's survival outcome (Cancer Genome Atlas Network 2012; Curtis et al. 2012; Vaske et al. 2010).

Finally, NGS supports the discovery of novel biomarkers such as mutation signatures and tumor mutational burden (TMB). Statistical analyses are performed, and patterns are discovered through millions of mutations detected by NGS. TMB has been shown to be an effective biomarker for predicting the response to immuno-therapy—an innovative area of research that can use the body's own immune system to fight cancer (Steuer and Ramalingam 2018). For all of these reasons, NGS has proven to be a powerful tool in clinical oncology. However, important challenges remain in cancer genomics and precision medicine fields such as efficiently leveraging the vast amount of genomic data available and making relevant treatment recommendations to clinicians. 


\section{Challenges in cancer genomics data interpretation}

Next-generation sequencing has revolutionized medical research and enabled multi-layer studies that integrate genomic data of high dimensionality such as DNA-seq, RNA-seq, and other multi-omics data such as proteome, epigenome, and microbiome. The integrative analysis of multi-omics data provides a more comprehensive view of biological processes leading to a better understanding of these systems compared to single-layer analysis (Chari et al. 2010; Wang et al. 2014).

However, there are several challenges to the translation of multi-omics data into clinically actionable biomarkers. First, combing data profiles at various levels would result in high dimensionality with large number of covariates. Data sparsity from high dimensionality combined with high heterogeneity from diverse types of data imposes a significant difficulty in integrative analyses. Many dimension reduction techniques such as multiple co-inertia analysis and multiple factor analysis have been developed to facilitate downstream joint analyses by mapping the data to lower dimensional space without a significant loss of information and transforming observations across data sets (Meng et al. 2016). Various integrating frameworks especially network-based approaches which use graphical algorithms to capture molecular network interactions and multi-level Bayesian models which impose realistic assumptions for parameter estimation through a prior-posterior Bayesian structure have been commonly applied in advanced strategies for multi-omics data analysis (Bersanelli et al. 2016).

Second, better standards for data generation and reporting are needed to facilitate data integration and to reduce bias (Ibrahim et al. 2016; Li et al. 2017). Sample acquisition and preparation procedures need to be well regulated for data generation and sequencing platform, and computational pipelines need to be carefully calibrated and validated. For instance, for NGS data, reference material (CLSI QMS01-A 2018; CLSI MM01A3E 2018; NIST 2018) whose properties are sufficiently homogeneous and well established to be used for the calibration of sequencing system is needed. The Centers for Disease Control (CDC) and Prevention's Genetic Testing Reference Material Coordination Program (GeT-RM) is engaging to generate renewable and publicly available characterized gDNA reference materials that can be used for clinical NGS testing. Other than reference materials, laboratory practice guidelines were published by CDC's Nex-StoCT II working group (Gargis et al. 2015). However, since hardware and software often get updated frequently and NGS analysis often encompass complex multi-step processes, further guidance for quality control criteria is needed, especially when sharing data among different laboratories. Those standards will help different laboratories to validate procedures, assess the quality of sequencing, evaluate performance of new platforms, and compare or share results among them.

Last, but not least, well-designed studies with causal inference are needed to filter out biomarkers that have strong correlative effects but no real causative effects in tumorigenesis (Ibrahim et al. 2016; MacArthur et al. 2014). Multiple classes of evidence may contribute to the pathogenic inference, including genetic, informatic, and experimental data. On the genetic level, the pathogenic variants could be significantly enriched in cases compared to controls and/ or the variant is co-inherited with disease status within affected families. On the informatic level, the pathogenic variants could be found at the location predicted to cause functional disruption (for example, protein-binding region). And on the experimental level, the pathogenic variants could significantly alter levels, splicing, or normal biochemical function of the product of the affected genes. This can be shown either in patient cells or well validated with in vitro or in vivo models such as introduction of the variant or an engineered gene product carrying the variant into cell lines or animal models results in phenotype consistent with the disease. Finally, the cellular phenotype in patient-derived cells, model organisms, or engineered equivalents can be rescued by addition of wild-type gene product or specific knockdown of the variant allele (MacArthur et al. 2014). Careful attention should be drawn on these aspects in regard to evaluating pathogenicity of new discovered biomarkers from omics data.

The advancement of ML technologies is bound to impact the interpretation of genomic sequencing data, which has traditionally relied on manual curation by experts in the field. These curation efforts rely on protein structure, functional studies and more recently, on "in silico" models that predict the functional impact of genetic alteration such as SIFT, PANTHER-PSEP, PolyPhen2, and others (Tang and Thomas 2016). Genomic databases such as ClinVar or COSMIC have proliferated as means of concisely compiling a collection of classified genetic variants. They provide the evidence supporting the classification of a variant as being pathogenic, benign or of unknown significance (VUS).

Two key limitations of manually curating and interpreting the results from genomics data are scalability and reproducibility. These challenges continue to grow as more genomic data become available. The number of curation experts or variant scientists and the amount of time that they can dedicate daily to this task is limited. Different variant scientists among companies, research groups, and hospitals can introduce bias due to subjectivity in curation criteria, adherence to Standardized Operating Procedures and training. To 
address these limitations, organizations are working to build and standardize multi-step protocols for variant classification such as the American College of Medical Genetics and Genomics and the Association for Molecular Pathology (ACMG-AMP), who, in 2015, published a series of guidelines for the interpretation of germline genetic variants for genes causative of hereditary human disorders (Richards et al. 2015). These guidelines have been adopted, refined, and tested in multiple institutions for several genetic diseases including cancer, Marfan Syndrome, and diabetes among others (Amendola et al. 2016; Muino-Mosquera et al. 2018; Richards et al. 2015; Santana et al. 2017; Sukhai et al. 2016). Similarly, the International Society for Gastrointestinal Hereditary Tumors (InSiGHT) has developed a standardized classification scheme for variants occurring in genes associated with hereditary gastrointestinal tumors such as Lynch Syndrome (Thompson et al. 2014). More recently, ACMG and AMP in collaboration with the American Society of Clinical Oncology, and College of American Pathologists have published guidelines for the classification, annotation, interpretation, and reporting for somatic sequence variants in cancer (Li et al. 2017). Yet, the ability to scale NGS variant interpretation and to maintain strict quality control remains limited.

\section{Precision medicine and Al}

Precision medicine or personalized medicine tackles diseases by tailoring treatment based on genomic, lifestyle, and environmental characteristics of each patient. With precision medicine and the advancement of NGS, genomic profiles of patients have been increasingly used for risk prediction, disease diagnosis, and development of targeted therapies. Gene expression is an important part of the patients' genomic profiles, and interestingly, ML classification methods applied to gene expression data are not new. Historically, comprehensive gene expression analysis was done with microarrays and now with RNA-seq. Expression data are analyzed to identify the significant genes in the upregulated or downregulated pathways (Lyu and Haque 2018; Hwang et al. 2002), and are also trained to predict the cancer subtypes and prognosis when outcome data or diagnosis information is available (Bartsch et al. 2016; Pepke and Ver Steeg 2017). Multiple review papers have already covered different ML applications on gene expression data (Molla et al. 2004; Sajda 2006; Kourou et al. 2014; Libbrecht 2015; Bashiri et al. 2017; Noor and Narwal 2017). In our review, we, however, will focus on AI applications related to NGS and cancer genomics testing (Fig. 1).

\section{Variant calling}

Variant calling is the process to identify variants in NGS data. Raw sequencing reads are first aligned to the reference genome and then run through multiple quality improvement steps to prepare for the variant calling (e.g., quality evaluation, recalibration, indel realignment, and identifying duplicates). Randomness of DNA molecule selection at the enrichment step, platform-dependent systematic errors, sequencing errors, and alignment errors constitute the main challenges of this step.

Out-of-box usage of most variant callers is not ideal, especially in a clinical setting. Often, users need to

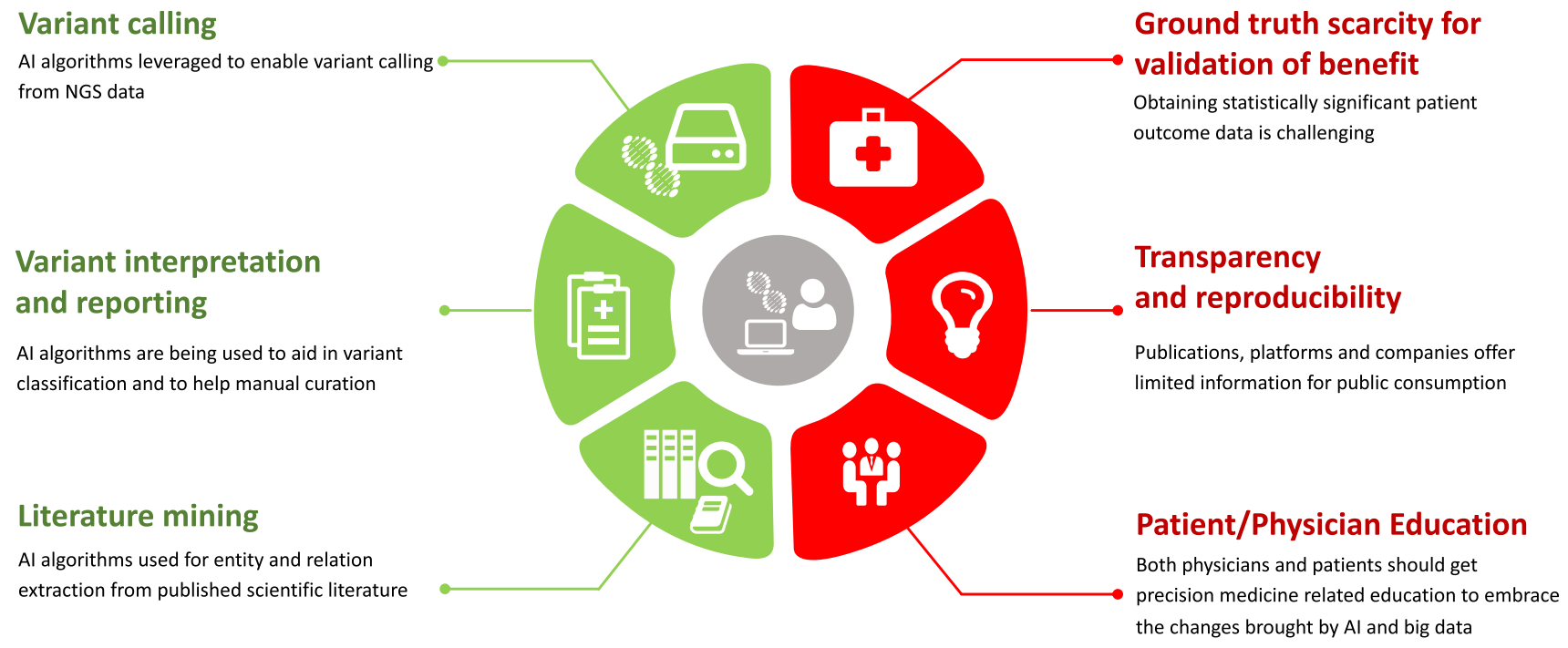

Fig. 1 Topics discussed in the review paper. This figure demonstrates that several key topics discussed in the paper with the green icons representing benefits or improvements and red icons representing challenges or caveats 
heuristically tune parameters and apply multiple customized filters to remove false positives before an acceptable accuracy is achieved. This is a time-consuming effort that requires expertise to fine tune quality scores and attributes within contexts of sequencing, amplicon, alignment, and genomics.

Different groups are now leveraging ML algorithms and training on those underlying quality features such as sequencing and alignment quality to improve the performance of the variant calling, especially in sub-optimal scenarios (Ding et al. 2012; Hao et al. 2017; Hill et al. 2018; Spinella et al. 2016). Tumor ploidy and purity are two major factors that contribute to cancer complexity. Sub-clonal variants (present only in a few cells) are difficult to detect, because their representation in the sequencing library is low. This can result in variability across analysis methods, thresholds, and quality scores which may not be flexible enough to detect sub-clonal variants. Instead of setting up static rules, ML methods are able to adjust the thresholds dynamically based on the patterns. Variants with very low allele frequencies can still be reported if the sequencing depth and other quality metrics outperform and pass the overall confidence threshold. For instance, a convolutional neural network $(\mathrm{CNN})$ model of which the algorithms are often used in image recognition achieved F1 score of 0.96 , and was able to reach variants with allele frequency as low as 0.0001 (Hill et al. 2018). F1 score is an accuracy measure that takes into account both precision and recall. In another instance, a Random Forest-based ML approach (Cerebro) applied to NGS data showed improved accuracy, as measured by F1 score, in the identification of tumor mutations when compared to the existing variant calling programs such as MuTect1, MuTect2, SomaticSniper, Strelka, VarDict, and VarScan2. While their recall values are fairly similar, Cerebro showed increased precision values comparing to the other methods (Wood et al. 2018). Similar successes of ML have been described in copy-number variation (CNV) analyses (Antaki et al. 2018; Onsongo et al. 2016).

Besides standard variant detection paradigms, Google's DeepVariant transforms a variant calling problem into an image recognition problem by converting a BAM file into images similar to genome browser snapshots and calls the variants based on likelihoods, using the Inception Tensor Flow framework which was originally developed for image classification (Going Deeper with Convolutions 2014). Another recent study successfully applied ML on sequencing data from multiple regions of a tumor to identify and learn growth patterns as accurate predictors for tumor progression (Caravagna et al. 2018).

\section{Variant interpretation and reporting}

Variant annotation and classification are the basis of genetic diagnostics and are crucial to clinical patient care and treatment planning. In vivo or in vitro functional studies are considered the gold standard for determining whether a mutation is benign or disease causing. Several computational methods have been applied for the identification of cancer driver mutations based on non-random distribution of mutations within proteins (Porta-Pardo et al. 2017). In silico prediction tools like PolyPhen and SIFT are widely used to assist the manual curation but have not established themselves as the determining factors in the clinical setting (Adzhubei et al. 2010; Vaser et al. 2016). Many research groups are training ML models on features encoding secondary structures, intrinsic disorders, DNA-binding, phosphorylation, conservation, predicted structure, and homolog counts to further improve the accuracy of variant classification, to incorporate high-dimensional data sets, and to unify the variant interpretation among laboratories. Some notable examples are deep neural networks (Bromberg et al. 2008; Ferrer-Costa et al. 2005; Qi et al. 2018; Quang et al. 2015), decision tree (Dobson et al. 2006; Krishnan and Westhead 2003), random forrest (Bao and Cui 2005; Carter et al. 2009; Kaminker et al. 2007; Li et al. 2009a; Wainreb et al. 2010), and support vector machine (Calabrese et al. 2009; Capriotti et al. 2006, 2008; Karchin et al. 2005; Yue and Moult 2006).

It is also imperative to evaluate and validate prediction tools. The critical assessment of genome interpretation (CAGI) has carried out prediction challenges accompanied with experimentally confirmed validated results throughout the years. The shared data and assessment publications are invaluable sources to set the standards for evaluation on the performance of any prediction tool. For example, the proprietary classification for BRCA mutations carried by Myriad Genetics has been considered an established assessment to evaluate pathogenicity in functional studies. Using ensemble learning methods on multimodal data sets, Pejaver et al. have developed missense variant pathogenicity predictors with high accuracy of predictions on BRCA missense variants classified by Myriad Genetics. Unfortunately, their evaluation is inconclusive due to small sample size, and only a small number of mutations were evaluated in this study (Pejaver et al. 2017). Saturation genome editing has been used in a recent study to assay all possible single-nucleotide variants in functionally critical domains of BRCA1 for variant functional classification and could be a viable strategy to overcome the challenge of lacking variant functional data, especially for those rare and low-frequency variants and to enable approaches with systematically derived measurements for functional analysis (Findlay et al. 2018).

Cancer biologists and molecular pathologists are trained to classify cancer sequence variants for their pathogenicity 
and clinical relevance. This is a complex process which is difficult to compile into a set of rules comprehensive enough to cover all scenarios. To what degree can ML algorithms learn the complex clinical decisions made by individual pathologists and classify the variants automatically? Massachusetts General Hospital (MGH) did the experiment and got very promising results. They selected $\sim 500$ features, built multiple ML models on $~ 20,000$ clinical sign-out variants reported by board-certified molecular pathologists and then compared the prediction results to find the best model (Zomnir et al. 2018). The logistic regression model demonstrated the best performance with only $1 \%$ false negativity and $2 \%$ false positivity, which is comparable to human decisions.

\section{Literature mining}

Owing to the open-access policies of many journals and the steady growth of scientific publications (Fig. 2), there is widespread availability of the published literature. PubMed currently comprises of over 28 million citations from Medline, life science journals, and online books (PubMed). The number of publications each year, as indexed in PubMed, has exceeded 1 million since 2011. This volume and veracity in publications indicate multiple hypotheses are being tested at the same time, which makes it harder for researchers to stay up to date in their field in the absence of some automated assists. It, therefore, impacts their ability to generate meaningful and coherent conclusions in a timely manner which are required for evidence-based recommendations in the context of precision medicine (Harmston et al. 2010). Applications and use of NLP-based solutions reduce the time and effort required for information retrieval (IR) and speed up curation, and provide novel opportunities for hypothesis generation based on the published literature (Caporaso et al. 2008; Extance 2018). In cancer genomics, publications per year can easily run into tens of thousands-far more than a researcher can keep up with - and this growth in publication has resulted in rapid growth of application of text mining and NLP techniques (Fig. 2). Biomedical named entity recognition (Bio-NER) and relationship extraction are two key NLP processes used in evidence extraction. The publicly available tools are reviewed and compared here (Table 1).

\section{Entity extraction}

Bio-NER is the foundation of evidence extraction for precision medicine. In cancer genomics, NLP tools have been used for the automated extraction of entities such as gene, genetic variants, treatments, and conditions. Identifying genetic variants is a key step for tumor molecular profiling and downstream gene-protein or gene-disease relationship analysis. Medical providers require the accurate identification and interpretation of genetic variation data to design effective personalized treatment strategies for their patients. Unfortunately, there is no universal standard for how genetic variants are called out and there are multiple ways of presenting the same event in the literature and genomic databases. Variation could be described at multiple description levels such as genomic and protein levels, and mapped against different reference genomes. Their mentions are often written as various natural language phrases besides the standard alphanumeric formats (HGVS format). To consolidate the knowledge on genetic variation from the literature mining and integrate it with curated data in existing resources such
Fig. 2 Publication number plotted against publication year. In this figure, two $y$-axes have been plotted. One $y$-axis represents the number for papers related to "Cancer Genomics". The other $y$-axis represents the number for papers related to "Cancer Genomics + NLP". The $x$-axis represents the publication year

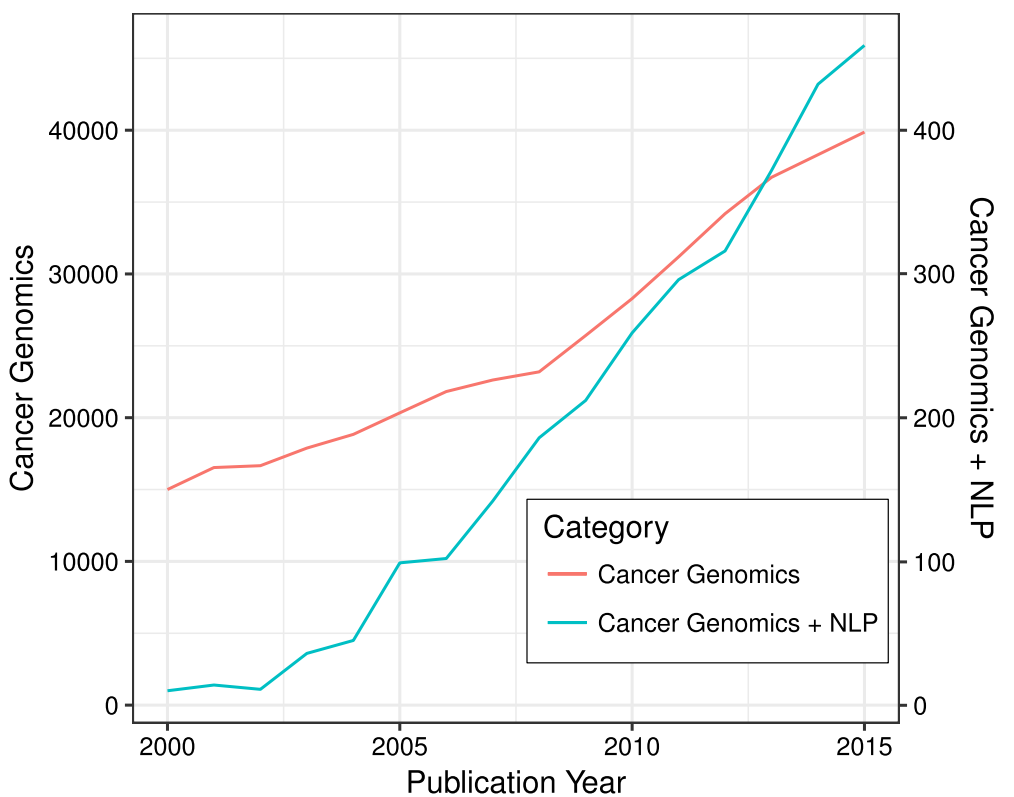




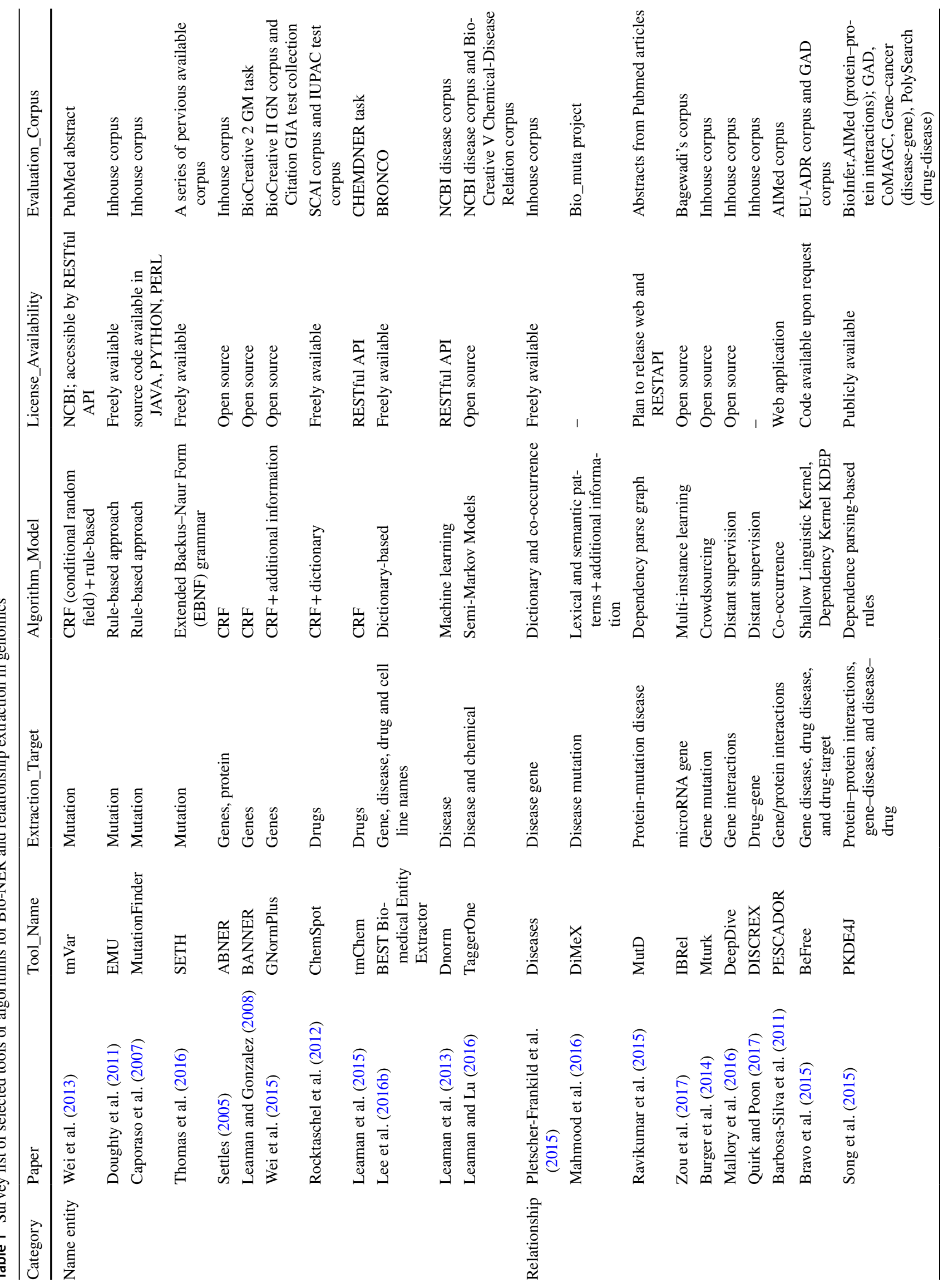




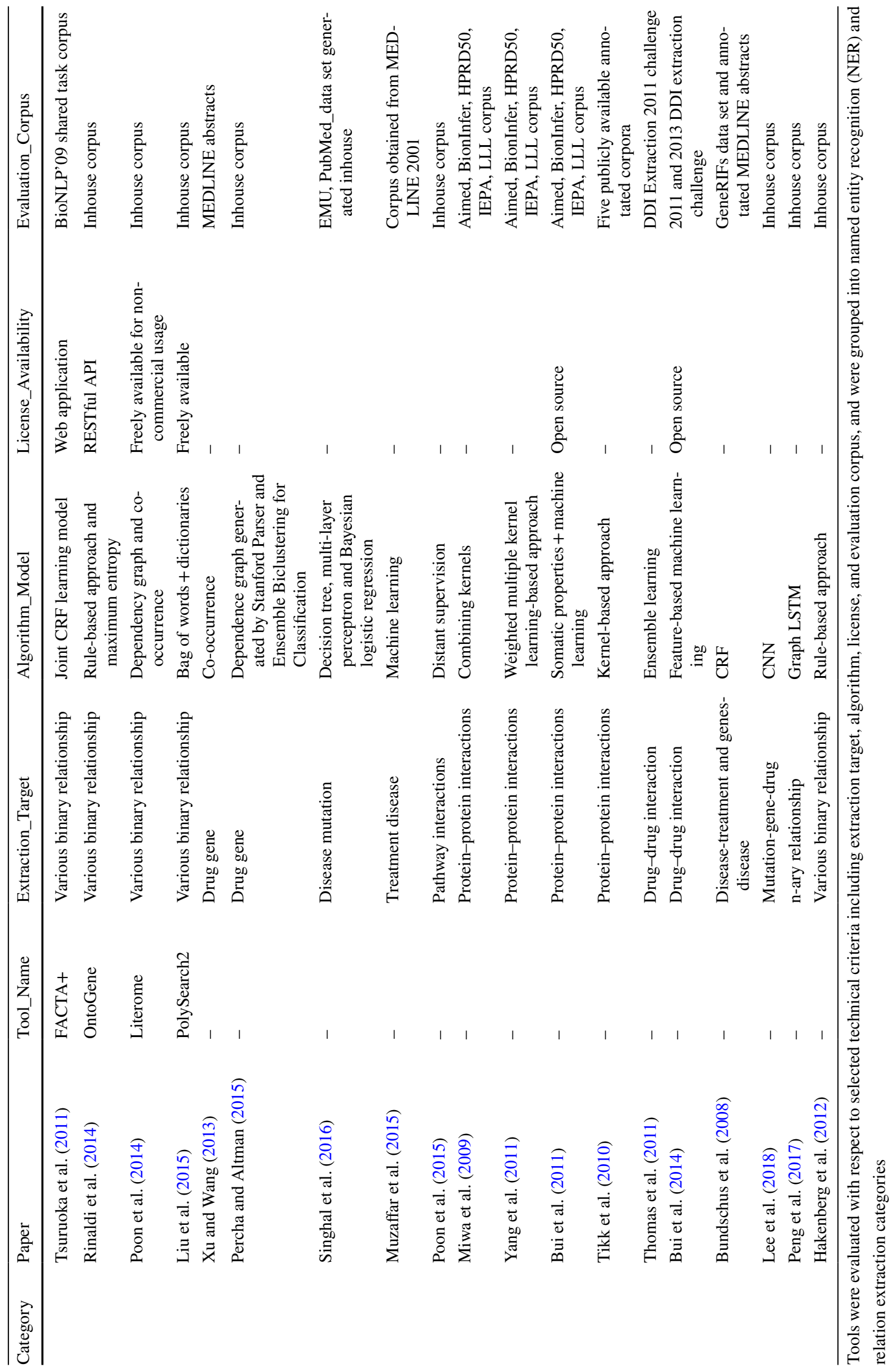


as ClinVar and COSMIC, it is essential to both standardize the genetic variations to HGVS nomenclature and normalize them to unique identifiers such as reference SNP ID number (RSIDs).

Current biomedical named entity recognition techniques fall into three major categories: dictionary-based approaches, rule-based approaches, and ML/deep learning approaches (Cohen and Hersh 2005; Li et al. 2009b). Dictionary-based approaches tend to be fast and simple but often miss undefined terms that are not mentioned in the dictionary, while rule-based approaches usually require handcrafted rules that identify terms from text but could be too specialized to adapt to new entity types (Rebholz-Schuhmann et al. 2011). ML approaches generally require the standard annotated training data sets for which the generation process is usually timeand labor-consuming (Krallinger et al. 2011). Recently, several deep learning methods have been applied to biomedical named entity recognition showing large performance gain by better integrating multi-dimensional features and, at the same time, minimizing manual feature generations (Habibi et al. 2017; Wu et al. 2017).

\section{Relationship extraction}

Relationships between recognized entities from the biomedical literature are key to identify the associations of genetic alterations, conditions, and treatments. These can be used as evidence for genetic test reporting by linking genotypeto-phenotype data, such as an association of a specific variant with drug sensitivity or of a variant with predisposition to a specific cancer type. The most intuitive and fastest approach for relation extraction is co-occurrence analysis, which tends to achieve high recall but low precision (Cheng et al. 2008; Doughty et al. 2011; Lee et al. 2016a). A rulebased approach can achieve higher precision (Hakenberg et al. 2012), but defining those rules can be time-consuming and labor-intensive. Many more sophisticated learning methods have been developed over the last decade and they conceptually fall into three categories: the supervised learning approach, the unsupervised or semi-supervised learning approach, and the hybrid learning approach. Within the scope of supervised learning, several papers focused on feature-based approach (Rink et al. 2011; Xu et al. 2012), while several other papers mainly used kernel-based approach (Kim et al. 2015; Ma et al. 2015; Tikk et al. 2013; Yang et al. 2011). Supervised approaches commonly require expensive labeled training data and their feature engineering and kernel selection would be time-consuming. Unsupervised learning approaches, on the other hand, focus on learning inherent structure in data and do not require labeled training data (Alicante et al. 2016; Quan et al. 2014). Unsupervised learning approaches such as association mining can help to identify interesting associations. Since the field of cancer genomics is rapidly evolving, to come up with meaningful evidence-based recommendations, it is important to make sense of vast available data sets and publications. Association mining has been used to identify frequently cooccurring entities to develop meaningful conclusions and recommendations (Alves et al. 2009). Other unsupervised technique such as clustering is used to develop insights into cancer signatures from multi-omics data. Semi-supervised learning such as the distant supervision approach usually utilize weakly labeled data derived from a knowledge base, which has been explored in cancer research (Quirk and Poon 2017). Hybrid approaches usually integrate pattern, rules, domain knowledge, and learning-based methods together to build models (Muzaffar et al. 2015). Deep learning integrates both supervised and unsupervised features by applying multi-layer non-linear functions for analysis and classification. Over recent years, deep learning methods like CNNs (Lee et al. 2018) and recurrent neural networks (RNNs) (Peng et al. 2017) have been applied into the relation extraction field and have led to promising results. Lee et al. demonstrated that $\mathrm{CNN}$ can be used for sentence-level relation classification. With features combining word embedding, type embedding, and position embedding, they achieved F1 score of 0.954 and 0.845 for mutation-gene and mutation-drug relationship classification, respectively, without explicitly defining keywords for relation extraction. Peng et al. showed that, by applying graph LSTM for drug-gene-mutation ternary relation extraction, they achieved precision 0.75 (with output probability threshold 0.9 ) in cross-sentence setting. In addition, graph LSTM outperformed a well-engineered feature-based classifier in extracting genetic pathway interactions using GENIA Event Extraction data set, illustrating its advantage of handling sparse linguistic patterns without intense feature engineering.

\section{Challenges to $\mathrm{Al}$ adoption in healthcare}

\section{Lack of ground truth to validate the benefit}

The evaluation of AI accuracy is critical to help gauge how well the system performs in assisting experts, and to make AI less of a black box. In cancer genomics, variant classification, clinical relevance, literature validation, and summarization are traditionally done by human experts. To prove the usefulness of an AI application, it needs to be evaluated in comparison with human experts and not only with the other AI solutions. However, this is rarely done due to the lack of publicly accessible knowledge bases for ground truth data.

Increasingly abundant patient genomic and clinical data generated from various genomic testing platforms are enabling AI solutions to discover novel clinically relevant patient subgroups for better clinical outcome (Kristensen 
Table 2 Major functionalities and transparency for key players in text mining and personalized medicine field

\begin{tabular}{|c|c|c|}
\hline Players & Functionality & Transparency \\
\hline Blueprint genetics & $\begin{array}{l}\text { Offers single gene test, targeted variant testing or whole } \\
\text { exome sequencing service along with interpretation }\end{array}$ & No explicit AI description \\
\hline Cambridge cancer genomics & Uses blood tests to guide cancer therapy & No explicit AI description \\
\hline Deep gene & $\begin{array}{l}\text { Provides cancer-type classifier based on deep learning } \\
\text { and somatic point mutations }\end{array}$ & Publication is available (Yuan et al. 2016) \\
\hline Deep genomics & $\begin{array}{l}\text { Develops genetic medicines using artificial intelligence } \\
\text { technology, with a focus on the preclinical develop- } \\
\text { ment of oligonucleotide therapies }\end{array}$ & $\begin{array}{l}\text { No detailed explanation but related publication is avail- } \\
\text { able (Wainberg et al. 2018) }\end{array}$ \\
\hline DeepVariant & $\begin{array}{l}\text { Analysis pipeline using a deep neural network to call } \\
\text { genetic variants from NGS DNA data }\end{array}$ & $\begin{array}{l}\text { Available in GitHub https://github.com/google/deepv } \\
\text { ariant }\end{array}$ \\
\hline Genomenon & $\begin{array}{l}\text { Genomic search engine and database to provide disease- } \\
\text { gene-variant relationships from the full text of the } \\
\text { scientific literature for gene and variant interpretation }\end{array}$ & No explicit AI description \\
\hline Genoox & $\begin{array}{l}\text { Fully customized platform for genetic applications } \\
\text { including primary, secondary and tertiary analyses }\end{array}$ & $\begin{array}{l}\text { No explicit AI description but related publications } \\
\text { available (Stajkovska et al. 2018) }\end{array}$ \\
\hline Literome & $\begin{array}{l}\text { Automatic curation system to extract genomic knowl- } \\
\text { edge from PubMed articles to facilitate browsing, } \\
\text { searching, and reasoning }\end{array}$ & Publications are available (Poon et al. 2014, 2015) \\
\hline Perthera & $\begin{array}{l}\text { Manage process from tumor testing through Perthera } \\
\text { Report to provide cancer patients and physicians } \\
\text { with therapeutic options ranked by the probability of } \\
\text { outcome }\end{array}$ & No explicit AI description \\
\hline Sophia Genetics & $\begin{array}{l}\text { Provides NGS data analysis to detect, annotate and } \\
\text { pre-classify genomic variants associated to multiple } \\
\text { disorder areas }\end{array}$ & No explicit AI description \\
\hline Watson for Genomics & $\begin{array}{l}\text { Provides in-depth clinical interpretation of the genetic } \\
\text { alterations in the sample automatically, enabling clini- } \\
\text { cal decision-making for personalized cancer care }\end{array}$ & $\begin{array}{l}\text { No explicit AI description but related publication is } \\
\text { available (Patel et al. 2018) }\end{array}$ \\
\hline WuXi NextCODE & $\begin{array}{l}\text { Uses genomics to identify the underlying biology and } \\
\text { advance the scientific understanding of disease and } \\
\text { propel the next generation of transformative therapies }\end{array}$ & $\begin{array}{l}\text { No explicit AI description but related publication is } \\
\text { available (Zhang et al. 2018) }\end{array}$ \\
\hline
\end{tabular}

For each company, the main functionality and transparency are summarized

et al. 2014; Kalinin et al. 2018). However, the difficulty of getting a statistically significant patient outcome data is one of the most pressing challenges to achieve an impactful solution. Patient outcome data are personal health information (PHI) that must be protected by the HIPAA guidelines in the US and GDPR in Europe. Given such regulations, sharing such data is not done lightly, as security considerations are vital to preventing sensitive data from being compromised (General Data Protection Regulation 2016; The Health Insurance Portability and Accountability Act of 1996 2014). Furthermore, there is a scarcity of patient outcome data available to be used in training and evaluation of AI systems for guiding the decisions of clinicians and experts in the design of treatment plans.

\section{Transparency and reproducibility}

$\mathrm{AI}$ is a hot field and its use has been claimed by many platforms and companies. However, detailed information on AI techniques and models is not clearly presented and there is considerable variability in methodologies from company to company. Based on publicly accessible information, we classify five different levels of transparency with a list of examples (Table 2).

Reproducibility of experimental results is the hallmark of science. Therefore, being able to replicate ML results and experiments is paramount. Because ML algorithms typically have lots of tunable components, performance can be affected by the sensitivity to the scale and quality of training data, empirical setting of hyperparameters, and initialization and optimization processes. Many publications fail to disclose simplifying assumptions or implementation details, and thus make it hard to reproduce results. This coupled with the fact that researchers often do not share their source code makes reproducibility a major challenge. Even if all details were shared, reproducibility is not easy to implement, as this requires that we either expect reviewers (a) to very carefully study the code and scripts needed to produce the results or (b) to create a new script based on the description of the algorithm and parameters in the paper. Simply running the 
scripts and checking whether the tables and graphs of the paper can be reproduced would do little to validate the work. These discussions promote the publication of well-described research methods and protocols, and thus help the advancement and adoption of AI technologies (Hutson 2018). Even after such hurdles are overcome, large-scale deployment of AI solutions in healthcare may happen only when benefits are realized via closely monitored and formally tested assessment in real world.

\section{Patient/physician education}

Digitization of healthcare has provided the access to bigdata information and cognitive insights to both caregivers and patients, transforming healthcare and clinical workflows (Mesko et al. 2017). The point-of-care has shifted from the clinic and physician to the patient. The old paradigm of paternalistic physician-patient relationship has been transformed into an equal-level partnership with shared medical decision-making. Experience-based medicine has evolved into evidence-based and patient-centered approaches. Both physicians and patients need to be prepared for this revolutionary role of AI in healthcare (Mesko et al. 2017).

Medical professionals must learn how to work alongside data-enabled technology applications and acquire knowledge about how AI works for healthcare delivery. Precision medicine relies on an increasing amount of heterogeneous data of molecular genetics, clinical, and biological parameters for each patient. The total number of parameters for medical decision-making on a single patient could be up to 10,000 by 2020 (Abernethy et al. 2010). It becomes impossible for a physician to bear with all the responsibilities of data management and analysis, not to mention patient communications. Physician burnout has become a pressing health challenge. The application of AI in healthcare aims to advise clinicians with better and faster insights to ultimately improve the lives of patients. By embracing AI, clinical teams could be relieved from repetitive daily work and have more time to focus on the other aspects of patient care (Fogel and Kvedar 2018). For instance, in one study, Watson for Genomics identified genomic alterations with potential clinical impact that were not recognized by the traditional molecular tumor boards in 323 (32\%) of patients using an analysis that took only a few minutes (Patel et al. 2018). At MGH, the clinical implementation of an AI-based decision support tool for variant reporting allows molecular pathologists to quickly make decisions and empowers them to explore the underlying reasoning behind them (Zomnir et al. 2018). As we move to an age of AI, medical education must move beyond the foundational biomedical and clinical sciences to knowledge of information platforms and intelligence tools in healthcare and the skills to effectively use them (Wartman and Combs 2018).
On the other hand, capturing data on individual variability in genomic, lifestyle, and clinical factors is at the core of precision medicine, which would empower patients to be more engaged in their health care. With augmented direct access to health and innovative technologies, transparency in healthcare would be improved and may lead to enhanced accountability and productivity. However, at the same time, the risk of patients getting exposed to unreliable or misinterpreted information and turning to non-validated and unregulated technological solutions is increasing (Mesko et al. 2017). To facilitate patient participation in this AIempowered digital health transformation, medical professionals should provide robust patient education initiatives related to precision medicine, benefits and risks of AI, data sharing, and protection. Healthcare providers need to be sensitive to varying degrees of patient preferences for privacy and properly obtain consent for patient data collection and use. The awareness of patients' rights and health literacy should be promoted to help patients navigate the modern technology-intensive healthcare system and to become accustomed to shared decision-making. Ethical principles should be developed to help ensure that development and use of AI applications, specifically within healthcare, are accurate, understandable, and beneficial.

\section{Perspectives: 5 years down the road}

We have entered the advent of an era in which AI can help across the medical continuum from research to prognosis, therapy, and post cancer treatment care. AI will remain the main driver to healthcare transformation towards precision medicine. While digital health has become essential for providing best practice in healthcare, it raises some unprecedented challenges (Bibault et al. 2016; Mesko et al. 2017). How this revolutionary role of $\mathrm{AI}$ in healthcare translates into an improvement in the actual lives of patients remains to be demonstrated and will be dependent on the availability of patient outcome data. More and more crowd-source challenges will be uniquely designed for problems in cancer genomics with experimentally defined ground truth to objectively and transparently evaluate the accuracy (CAGI 2018; Dream Challenges 2018; Grant Challenges 2018; PrecisionFDA True Challenge 2018). The data sets that will get published following these efforts will help to establish the standards for benchmarking and testing novel algorithms in the cancer community. Data protection, data sharing, and international standardization will be addressed and regulated (Center for Data Innovation). All those unprecedented challenges digital health poses should be addressed to ensure AI safety and beneficial impact to healthcare. 


\section{Compliance with ethical standards}

Conflict of interest The authors are employees of IBM Watson Health.

OpenAccess This article is distributed under the terms of the Creative Commons Attribution 4.0 International License (http://creativeco mmons.org/licenses/by/4.0/), which permits unrestricted use, distribution, and reproduction in any medium, provided you give appropriate credit to the original author(s) and the source, provide a link to the Creative Commons license, and indicate if changes were made.

\section{References}

Abadi S, Yan WX, Amar D, Mayrose I (2017) A machine learning approach for predicting CRISPR-Cas9 cleavage efficiencies and patterns underlying its mechanism of action. PLoS Comput Biol 13:e1005807. https://doi.org/10.1371/journal.pcbi.1005807

Abernethy AP, Etheredge LM, Ganz PA, Wallace P, German RR, Neti C, Bach PB, Murphy SB (2010) Rapid-learning system for cancer care. J Clin Oncol 28:4268-4274. https://doi.org/10.1200/ JCO.2010.28.5478

Adzhubei IA, Schmidt S, Peshkin L, Ramensky VE, Gerasimova A, Bork P, Kondrashov AS, Sunyaev SR (2010) A method and server for predicting damaging missense mutations. Nat Methods 7:248-249. https://doi.org/10.1038/nmeth0410-248

Alicante A, Corazza A, Isgro F, Silvestri S (2016) Unsupervised entity and relation extraction from clinical records in Italian. Comput Biol Med 72:263-275. https://doi.org/10.1016/j.compbiomed .2016 .01 .014

Alves R, Rodriguez-Baena DS, Aguilar-Ruiz JS (2009) Gene association analysis: a survey of frequent pattern mining from gene expression data. Brief Bioinform 11:210-224. https://doi. org/10.1093/bib/bbp042

Amendola LM et al (2016) Performance of ACMG-AMP variantinterpretation guidelines among nine laboratories in the clinical sequencing exploratory research consortium. Am J Hum Genet 99:247. https://doi.org/10.1016/j.ajhg.2016.06.001

Antaki D, Brandler WM, Sebat J (2018) SV2: accurate structural variation genotyping and de novo mutation detection from whole genomes. Bioinformatics 34:1774-1777. https://doi.org/10.1093/ bioinformatics/btx 813

Aravanis AM, Lee M, Klausner RD (2017) Next-generation sequencing of circulating tumor DNA for early cancer detection. Cell 168(4):571-574. https://doi.org/10.1016/j.cell.2017.01.030

Bao L, Cui Y (2005) Prediction of the phenotypic effects of non-synonymous single nucleotide polymorphisms using structural and evolutionary information. Bioinformatics 21:2185-2190. https ://doi.org/10.1093/bioinformatics/bti365

Barbosa-Silva A, Fontaine JF, Donnard ER, Stussi F, Ortega JM, Andrade-Navarro MA (2011) PESCADOR, a webbased tool to assist text-mining of biointeractions extracted from PubMed queries. BMC Bioinform 12:435. https://doi. org/10.1186/1471-2105-12-435

Bartsch G Jr et al (2016) Use of artificial intelligence and machine learning algorithms with gene expression profiling to predict recurrent nonmuscle invasive urothelial carcinoma of the bladder. J Urol 195(2):493-498. https://doi.org/10.1016/j. juro.2015.09.090

Bashiri A, Ghazisaeedi M, Safdari R, Shahmoradi L, Ehtesham H (2017) Improving the prediction of survival in cancer patients by using machine learning techniques: experience of gene expression data: a narrative review. Iran J Public Health 46(2):165-172
Bedi $G$ et al (2015) Automated analysis of free speech predicts psychosis onset in high-risk youths. NPJ Schizophr 1:15030. https ://doi.org/10.1038/npjschz.2015.30

Bersanelli M, Mosca E, Remondini D, Giampieri E, Sala C, Castellani G, Milanesi L (2016) Methods for the integration of multi-omics data: mathematical aspects. BMC Bioinform 17(Suppl 2):15 https://doi.org/10.1186/s12859-015-0857-9

Bibault JE, Giraud P, Burgun A (2016) Big Data and machine learning in radiation oncology: State of the art and future prospects. Cancer Lett 382:110-117. https://doi.org/10.1016/j. canlet.2016.05.033

Bravo A, Pinero J, Queralt-Rosinach N, Rautschka M, Furlong LI (2015) Extraction of relations between genes and diseases from text and large-scale data analysis: implications for translational research. BMC Bioinform 16:55. https://doi.org/10.1186/s1285 9-015-0472-9

Bromberg Y, Yachdav G, Rost B (2008) SNAP predicts effect of mutations on protein function. Bioinformatics 24:2397-2398. https://doi.org/10.1093/bioinformatics/btn435

Bui QC, Katrenko S, Sloot PM (2011) A hybrid approach to extract protein-protein interactions. Bioinformatics 27:259-265. https ://doi.org/10.1093/bioinformatics/btq620

Bui QC, Sloot PM, van Mulligen EM, Kors JA (2014) A novel feature-based approach to extract drug-drug interactions from biomedical text. Bioinformatics 30:3365-3371. https://doi. org/10.1093/bioinformatics/btu557

Bundschus M, Dejori M, Stetter M, Tresp V, Kriegel HP (2008) Extraction of semantic biomedical relations from text using conditional random fields. BMC Bioinform 9:207. https://doi. org/10.1186/1471-2105-9-207

Burger JD et al (2014) Hybrid curation of gene-mutation relations combining automated extraction and crowdsourcing. Database (Oxford). https://doi.org/10.1093/database/bau094

CAGI (2018) https://genomeinterpretation.org/. Accessed 10 Sept 2018

Calabrese R, Capriotti E, Fariselli P, Martelli PL, Casadio R (2009) Functional annotations improve the predictive score of human disease-related mutations in proteins. Hum Mutat 30:1237-1244. https://doi.org/10.1002/humu.21047

Cancer Genome Atlas Network (2012) Comprehensive molecular portraits of human breast tumours. Nature 490(7418):61-70. https:// doi.org/10.1038/nature11412

Cancer Genome Atlas Research Network, Weinstein JN, Collisson EA, Mills GB, Shaw KR, Ozenberger BA, Ellrott K, Shmulevich I, Sander C, Stuart JM (2013) The Cancer Genome Atlas PanCancer analysis project. Nat Genet 45(10):1113-1120. https:// doi.org/10.1038/ng.2764

Caporaso JG, Baumgartner WA Jr, Randolph DA, Cohen KB, Hunter L (2007) MutationFinder: a high-performance system for extracting point mutation mentions from text. Bioinformatics 23:18621865. https://doi.org/10.1093/bioinformatics/btm235

Caporaso JG, Deshpande N, Fink JL, Bourne PE, Cohen KB, Hunter L (2008) Intrinsic evaluation of text mining tools may not predict performance on realistic tasks. In: Pac. Symp. Biocomput., pp 640-651

Capriotti E, Calabrese R, Casadio R (2006) Predicting the insurgence of human genetic diseases associated to single point protein mutations with support vector machines and evolutionary information. Bioinformatics 22:2729-2734. https://doi.org/10.1093/ bioinformatics/btl423

Capriotti E, Arbiza L, Casadio R, Dopazo J, Dopazo H, Marti-Renom MA (2008) Use of estimated evolutionary strength at the codon level improves the prediction of disease-related protein mutations in humans. Hum Mutat 29:198-204. https://doi.org/10.1002/ humu. 20628

Caravagna G, Giarratano Y, Ramazzotti D, Tomlinson I, Graham TA, Sanguinetti G, Sottoriva A (2018) Detecting repeated cancer 
evolution from multi-region tumor sequencing data. Nat Methods 15:707-714. https://doi.org/10.1038/s41592-018-0108-x

Carter $\mathrm{H}$ et al (2009) Cancer-specific high-throughput annotation of somatic mutations: computational prediction of driver missense mutations. Cancer Res 69:6660-6667. https://doi. org/10.1158/0008-5472.CAN-09-1133

Cava C, Bertoli G, Colaprico A, Olsen C, Bontempi G, Castiglioni I (2018) Integration of multiple networks and pathways identifies cancer driver genes in pan-cancer analysis. BMC Genom 19(1):25. https://doi.org/10.1186/s12864-017-4423-x

Chang EK et al (2016) Defining a patient population with cirrhosis: an automated algorithm with natural language processing. J Clin Gastroenterol 50:889-894. https://doi.org/10.1097/ MCG.0000000000000583

Chari R, Coe BP, Vucic EA, Lockwood WW, Lam WL (2010) An integrative multi-dimensional genetic and epigenetic strategy to identify aberrant genes and pathways in cancer. BMC Syst Biol 4:67. https://doi.org/10.1186/1752-0509-4-6

Cheng D, Knox C, Young N, Stothard P, Damaraju S, Wishart DS (2008) PolySearch: a web-based text mining system for extracting relationships between human diseases, genes, mutations, drugs and metabolites. Nucleic Acids Res 36:W399-W405. https://doi.org/10.1093/nar/gkn296

CLSI MM01A3E (2018) https://clsi.org/standards/products/molec ular-methods/documents/mm01/. Accessed 10 Sept 2018

CLSI QMS01-A (2018) https://clsi.org/standards/products/qualitymanagement-systems/documents/qms01/. Accessed $10 \mathrm{Sept}$ 2018

Cohen AM, Hersh WR (2005) A survey of current work in biomedical text mining. Brief Bioinform 6:57-71

Cohen JD et al (2018) Detection and localization of surgically resectable cancers with a multi-analyte blood test. Science 359:926930. https://doi.org/10.1126/science.aar3247

Curtis C, Shah SP, Chin SF, Turashvili G, Rueda OM, Dunning MJ, Speed D, Lynch AG, Samarajiwa S, Yuan Y, Gräf S, Ha G, Haffari G, Bashashati A, Russell R, McKinney S, METABRIC Group, Langerød A, Green A, Provenzano E, Wishart G, Pinder S, Watson P, Markowetz F, Murphy L, Ellis I, Purushotham A, Børresen-Dale AL, Brenton JD, Tavaré S, Caldas C, Aparicio S (2012) The genomic and transcriptomic architecture of 2,000 breast tumours reveals novel subgroups. Nature 486(7403):346-352. https://doi.org/10.1038/nature10983

Davis RJ, Gönen M, Margineantu DH, Handeli S, Swanger J, Hoellerbauer P, Paddison PJ, Gu H, Raftery D, Grim JE, Hockenbery DM, Margolin AA, Clurman BE (2018) Pan-cancer transcriptional signatures predictive of oncogenic mutations reveal that Fbw7 regulates cancer cell oxidative metabolism. Proc Natl Acad Sci USA 115(21):5462-5467. https://doi. org/10.1073/pnas. 1718338115

De Fauw J et al (2018) Clinically applicable deep learning for diagnosis and referral in retinal disease. Nat Med. https://doi. org/10.1038/s41591-018-0107-6

Ding J et al (2012) Feature-based classifiers for somatic mutation detection in tumour-normal paired sequencing data. Bioinformatics 28:167-175. https://doi.org/10.1093/bioinformatics/ btr629

Dobson RJ, Munroe PB, Caulfield MJ, Saqi MA (2006) Predicting deleterious nsSNPs: an analysis of sequence and structural attributes. BMC Bioinform 7:217. https://doi.org/10.1186/1471-2105-7-217

Doughty E, Kertesz-Farkas A, Bodenreider O, Thompson G, Adadey A, Peterson T, Kann MG (2011) Toward an automatic method for extracting cancer- and other disease-related point mutations from the biomedical literature. Bioinformatics 27:408-415. https ://doi.org/10.1093/bioinformatics/btq667

Dream Challenges (2018) http://dreamchallenges.org/project/. Accessed 10 Sept 2018
Ehteshami Bejnordi B et al (2017) Diagnostic assessment of deep learning algorithms for detection of lymph node metastases in women with breast cancer. JAMA 318:2199-2210. https://doi. org/10.1001/jama.2017.14585

Extance A (2018) How AI technology can tame the scientific literature. https://www.nature.com/articles/d41586-018-06617-5. Accessed 10 Sept 2018

Ferrer-Costa C, Gelpi JL, Zamakola L, Parraga I, de la Cruz X, Orozco M (2005) PMUT: a web-based tool for the annotation of pathological mutations on proteins. Bioinformatics 21:3176-3178. https://doi.org/10.1093/bioinformatics/bti486

Findlay GM, Daza RM, Martin B, Zhang MD, Leith AP, Gasperini M, Janizek JD, Huang X, Starita LM, Shendure J (2018) Accurate classification of BRCA1 variants with saturation genome editing. Nature 562(7726):217-222. https://doi.org/10.1038/s4158 6-018-0461-z

Fogel AL, Kvedar JC (2018) Artificial intelligence powers digital medicine. npj Dig Med. https://doi.org/10.1038/s41746-017-0012-2

FoundationOne CDx (2018) https://www.accessdata.fda.gov/scripts/ cdrh/cfdocs/cfpma/pma.cfm?id=P170019. Accessed 10 Sept 2018

Gargis et al (2015) Good laboratory practice for clinical next-generation sequencing informatics pipelines. Nat Biotechnol 33(7):689693. https://doi.org/10.1038/nbt.3237

Garvin JH et al (2018) Automating quality measures for heart failure using natural language processing: a descriptive study in the department of veterans affairs. JMIR Med Inform 6:e5. https:// doi.org/10.2196/medinform.9150

General Data Protection Regulation (2016) Pub. L119, 4 May 2016, pp 1-88. https://eugdpr.org/. Accessed 10 Sept 2018

Going Deeper with Convolutions (2014) arXiv:1409.4842v1

Grant Challenges (2018) https://grand-challenge.org/challenges/. Accessed 10 Sept 2018

Habibi M, Weber L, Neves M, Wiegandt DL, Leser U (2017) Deep learning with word embeddings improves biomedical named entity recognition. Bioinformatics 33:i37-i48. https://doi. org/10.1093/bioinformatics/btx228

Haenssle HA et al (2018) Man against machine: diagnostic performance of a deep learning convolutional neural network for dermoscopic melanoma recognition in comparison to 58 dermatologists. Ann Oncol 29:1836-1842. https://doi.org/10.1093/annonc/mdy166

Hakenberg J et al (2012) A SNPshot of PubMed to associate genetic variants with drugs, diseases, and adverse reactions. J Biomed Inform 45:842-850. https://doi.org/10.1016/j.jbi.2012.04.006

Hao Y, Xuei X, Li L, Nakshatri H, Edenberg HJ, Liu Y (2017) RareVar: a framework for detecting low-frequency single-nucleotide variants. J Comput Biol 24:637-646. https://doi.org/10.1089/ cmb.2017.0057

Harmston N, Filsell W, Stumpf MP (2010) What the papers say: text mining for genomics and systems biology. Hum Genom 5:17-29

Hill ST et al (2018) Deep learning mutation prediction enables early stage lung cancer detection in liquid biopsy. IN: ICLR 2018 conference, Vancouver

Hutson M (2018) Missing data hinder replication of artificial intelligence studies. http://www.sciencemag.org/news/2018/02/ missing-data-hinder-replication-artificial-intelligence-studies. Accessed 10 Sept 2018

Hwang KB, Cho DY, Park SW, Kim SD, Zhang BT (2002) Applying machine learning techniques to analysis of gene expression data: cancer diagnosis. In: Lin SM, Johnson KF (eds) Methods of microarray data analysis, pp 167-182

Ibrahim R, Pasic M, Yousef GM (2016) Omics for personalized medicine: defining the current we swim in. Expert Rev Mol Diagn 16:719-722. https://doi.org/10.1586/14737159.2016.1164601

Kalinin AA et al (2018) Deep learning in pharmacogenomics: from gene regulation to patient stratification. Pharmacogenomics 19:629-650. https://doi.org/10.2217/pgs-2018-0008 
Kaminker JS, Zhang Y, Watanabe C, Zhang Z (2007) CanPredict: a computational tool for predicting cancer-associated missense mutations. Nucleic Acids Res 35:W595-W598. https://doi. org/10.1093/nar/gkm405

Karchin R et al (2005) LS-SNP: large-scale annotation of coding nonsynonymous SNPs based on multiple information sources. Bioinformatics 21:2814-2820. https://doi.org/10.1093/bioinforma tics/bti442

Kim S, Liu H, Yeganova L, Wilbur WJ (2015) Extracting drug-drug interactions from literature using a rich feature-based linear kernel approach. J Biomed Inform 55:23-30. https://doi. org/10.1016/j.jbi.2015.03.002

Kourou K, Exarchos TP, Exarchos KP, Karamouzis MV, Fotiadis DI (2014) Machine learning applications in cancer prognosis and prediction. Comput Struct Biotechnol J 13:8-17. https://doi. org/10.1016/j.csbj.2014.11.005 (eCollection 2015. Review)

Krallinger M et al (2011) The Protein-Protein Interaction tasks of BioCreative III: classification/ranking of articles and linking bioontology concepts to full text. BMC Bioinform 12(Suppl 8):S3. https://doi.org/10.1186/1471-2105-12-S8-S3

Krishnan VG, Westhead DR (2003) A comparative study of machinelearning methods to predict the effects of single nucleotide polymorphisms on protein function. Bioinformatics 19:2199-2209

Kristensen VN, Lingjærde OC, Russnes HG, Vollan HK, Frigessi A, Børresen-Dale AL (2014) Principles and methods of integrative genomic analyses in cancer. Nat Rev Cancer 14(5):299-313. https://doi.org/10.1038/nrc3721

Leaman R, Gonzalez G (2008) BANNER: an executable survey of advances in biomedical named entity recognition. In: Pac. Symp. Biocomput., pp 652-663

Leaman R, Lu Z (2016) TaggerOne: joint named entity recognition and normalization with semi-Markov Models. Bioinformatics 32:2839-2846. https://doi.org/10.1093/bioinformatics/btw343

Leaman R, Islamaj Dogan R, Lu Z (2013) DNorm: disease name normalization with pairwise learning to rank. Bioinformatics 29:2909-2917. https://doi.org/10.1093/bioinformatics/btt474

Leaman R, Wei CH, Lu Z (2015) tmChem: a high performance approach for chemical named entity recognition and normalization. $\mathbf{J}$ Cheminform 7:S3. https://doi.org/10.1186/1758-2946-7-S1-S3

Lee K et al (2016a) HiPub: translating PubMed and PMC texts to networks for knowledge discovery. Bioinformatics 32:2886-2888. https://doi.org/10.1093/bioinformatics/btw511

Lee $S$ et al (2016b) BEST: next-generation biomedical entity search tool for knowledge discovery from biomedical literature. PLoS One 11:e0164680. https://doi.org/10.1371/journal.pone.0164680

Lee K et al (2018) Deep learning of mutation-gene-drug relations from the literature. BMC Bioinform 19:21. https://doi.org/10.1186/ s12859-018-2029-1

Li B et al (2009a) Automated inference of molecular mechanisms of disease from amino acid substitutions. Bioinformatics 25:27442750. https://doi.org/10.1093/bioinformatics/btp528

Li L, Zhou R, Huang D (2009b) Two-phase biomedical named entity recognition using CRFs. Comput Biol Chem 33:334-338. https ://doi.org/10.1016/j.compbiolchem.2009.07.004

Li MM et al (2017) Standards and guidelines for the interpretation and reporting of sequence variants in cancer: a joint consensus recommendation of the Association for Molecular Pathology, American Society of Clinical Oncology, and College of American Pathologists. J Mol Diagn 19:4-23. https://doi.org/10.1016/j. jmoldx.2016.10.002

Li Y, Shi W, Wasserman WW (2018) Genome-wide prediction of cis-regulatory regions using supervised deep learning methods. BMC Bioinform 19:202. https://doi.org/10.1186/s1285 9-018-2187-1

Libbrecht MW (2015) Machine learning in genetics and genomics. Nat Rev Genet 16(6):321-332. https://doi.org/10.1038/nrg3920
Lin E, Kuo PH, Liu YL, Yu YW, Yang AC, Tsai SJ (2018) A deep learning approach for predicting antidepressant response in major depression using clinical and genetic biomarkers. Front Psychiatry 9:290. https://doi.org/10.3389/fpsyt.2018.00290

Liu Y, Liang Y, Wishart D (2015) PolySearch2: a significantly improved text-mining system for discovering associations between human diseases, genes, drugs, metabolites, toxins and more. Nucleic Acids Res 43:W535-W542. https://doi. org/10.1093/nar/gkv383

Low SK, Zembutsu H, Nakamura Y (2018) Breast cancer: The translation of big genomic data to cancer precision medicine. Cancer Sci 109:497-506. https://doi.org/10.1111/cas.13463

Lyu B, Haque A (2018) Deep learning based tumor type classification using gene expression data. https://doi.org/10.1101/364323

Ma C, Zhang Y, Zhang M (2015) Tree Kernel-based Protein-Protein interaction extraction considering both modal verb phrases and appositive dependency features. In: Paper presented at the proceedings of the 24th international conference on world wide web-WWW' 15 companion

MacArthur DG et al (2014) Guidelines for investigating causality of sequence variants in human disease. Nature 508:469-476. https ://doi.org/10.1038/nature13127

Mahmood AS, Wu TJ, Mazumder R, Vijay-Shanker K (2016) DiMeX: a text mining system for mutation-disease association extraction. PLoS One 11:e0152725. https://doi.org/10.1371/journ al.pone. 0152725

Mallory EK, Zhang C, Re C, Altman RB (2016) Large-scale extraction of gene interactions from full-text literature using DeepDive. Bioinformatics 32:106-113. https://doi.org/10.1093/bioinforma tics/btv476

Meng C, Zeleznik OA, Thallinger GG, Kuster B, Gholami AM, Culhane AC (2016) Dimension reduction techniques for the integrative analysis of multi-omics data. Brief Bioinform 17:628-641. https://doi.org/10.1093/bib/bbv108

Mesko B, Drobni Z, Benyei E, Gergely B, Gyorffy Z (2017) Digital health is a cultural transformation of traditional healthcare. Mhealth 3:38. https://doi.org/10.21037/mhealth.2017.08.07

Meystre S, Haug PJ (2006) Natural language processing to extract medical problems from electronic clinical documents: performance evaluation. J Biomed Inform 39:589-599. https://doi. org/10.1016/j.jbi.2005.11.004

Miotto R, Li L, Kidd BA, Dudley JT (2016) Deep patient: an unsupervised representation to predict the future of patients from the electronic health records. Sci Rep 6:26094. https://doi. org/10.1038/srep26094

Miwa M, Saetre R, Miyao Y, Tsujii J (2009) Protein-protein interaction extraction by leveraging multiple kernels and parsers. Int J Med Inform 78:e39-e46. https://doi.org/10.1016/j.ijmed inf.2009.04.010

Molla M, Waddell M, Page D, Shavlik J (2004) Using machine learning to design and interpret gene-expression microarrays. AI Mag 25:23-44. https://doi.org/10.1609/aimag.v25i1.1745

Muino-Mosquera L et al (2018) Tailoring the American College of Medical Genetics and Genomics and the Association for Molecular Pathology Guidelines for the Interpretation of Sequenced Variants in the FBN1 Gene for Marfan Syndrome: proposal for a disease- and gene-specific guideline. Circ Genom Precis Med 11:e02039. https://doi.org/10.1161/CIRCGEN.117.002039

Muzaffar AW, Azam F, Qamar U (2015) A relation extraction framework for biomedical text using hybrid feature set. Comput Math Methods Med 2015:910423 https://doi.org/10.1155/2015/910423

NIST (2018) https://www.nist.gov/programs-projects/cancer-bioma rker-reference-materials Accessed 10 Sept 2018

Noor MM, Narwal V (2017) Machine learning approaches in cancer detection and diagnosis: mini review. https://doi.org/10.13140/ RG.2.2.27775.51363 
Onsongo G, Baughn LB, Bower M, Henzler C, Schomaker M, Silverstein KA, Thyagarajan B (2016) CNV-RF is a random forest-based copy number variation detection method using nextgeneration sequencing. J Mol Diagn 18:872-881. https://doi. org/10.1016/j.jmoldx.2016.07.001

Osborne JD, Wyatt M, Westfall AO, Willig J, Bethard S, Gordon G (2016) Efficient identification of nationally mandated reportable cancer cases using natural language processing and machine learning. J Am Med Inform Assoc 23:1077-1084. https://doi. org/10.1093/jamia/ocw006

Palmirotta R, Lovero D, Cafforio P, Felici C, Mannavola F, Pellè E, Quaresmini D, Tucci M, Silvestris F (2018) Liquid biopsy of cancer: a multimodal diagnostic tool in clinical oncology. Ther Adv Med Oncol 10:1758835918794630. https://doi. org/10.1177/1758835918794630

Patel NM et al (2018) Enhancing next-generation sequencing-guided cancer care through cognitive computing. Oncologist 23:179185. https://doi.org/10.1634/theoncologist.2017-0170

Pejaver V, Mooney SD, Radivojac P (2017) Missense variant pathogenicity predictors generalize well across a range of functionspecific prediction challenges. Hum Mutat 38:1092-1108. https ://doi.org/10.1002/humu.23258

Peng N, Poon H, Quirk C, Toutanova KWY (2017) Cross-sentence $\mathrm{N}$-ary relation extraction with graph LSTMs. arXiv:1708.03743 [cs.CL]

Pennell NA, Mutebi A, Zhou ZY (2018) Economic impact of next generation sequencing vs sequential single-gene testing modalities to detect genomic alterations in metastatic non-small cell lung cancer using a decision analytic model. In: ASCO

Pepke S, Ver Steeg G (2017) Comprehensive discovery of subsample gene expression components by information explanation: therapeutic implications in cancer. BMC Med Genom 10(1):12. https ://doi.org/10.1186/s12920-017-0245-6

Percha B, Altman RB (2015) Learning the structure of biomedical relationships from unstructured text. PLoS Comput Biol 11:e1004216. https://doi.org/10.1371/journal.pcbi.1004216

Pletscher-Frankild S, Palleja A, Tsafou K, Binder JX, Jensen LJ (2015) DISEASES: text mining and data integration of disease-gene associations. Methods 74:83-89. https://doi.org/10.1016/j.ymeth .2014.11.020

Poon H, Quirk C, DeZiel C, Heckerman D (2014) Literome: PubMedscale genomic knowledge base in the cloud. Bioinformatics 30:2840-2842. https://doi.org/10.1093/bioinformatics/btu383

Poon H, Toutanova K, Quirk C (2015) Distant supervision for cancer pathway extraction from text. In: Paper presented at the pacific symposium on biocomputing

Porta-Pardo E, Kamburov A, Tamborero D, Pons T, Grases D, Valencia A, Lopez-Bigas N, Getz G, Godzik (2017) Comparison of algorithms for the detection of cancer drivers at subgene resolution. Nat Methods 14(8):782-788. https://doi.org/10.1038/nmeth.4364

Praxis Extended RAS Panel (2018) https://www.accessdata.fda.gov/ scripts/cdrh/cfdocs/cfpma/pma.cfm?id=P160038. Accessed 10 Sept 2018

PrecisionFDA True Challenge (2018) https://precision.fda.gov/chall enges/truth. Accessed 10 Sept 2018

Qi H, Chen C, Zhang H, Long JJ, Chung WK, Guan Y, Shen Y (2018) MVP: predicting pathogenicity of missense variants by deep learning. https://doi.org/10.1101/259390

Quan C, Wang M, Ren F (2014) An unsupervised text mining method for relation extraction from biomedical literature. PLoS One 9:e102039. https://doi.org/10.1371/journal.pone.0102039

Quang D, Chen Y, Xie X (2015) DANN: a deep learning approach for annotating the pathogenicity of genetic variants. Bioinformatics 31:761-763. https://doi.org/10.1093/bioinformatics/btu703

Quirk C, Poon H (2017) Distant supervision for relation extraction beyond the sentence boundary. arXiv:1609.04873v3 [cs.CL]
Ravikumar KE, Wagholikar KB, Li D, Kocher JP, Liu H (2015) Text mining facilitates database curation-extraction of mutation-disease associations from Bio-medical literature. BMC Bioinform 16:185. https://doi.org/10.1186/s12859-015-0609-X

Rebholz-Schuhmann D et al (2011) Assessment of NER solutions against the first and second CALBC Silver Standard Corpus. J Biomed Semant 2(Suppl 5):S11 https://doi. org/10.1186/2041-1480-2-S5-S11

Richards S et al (2015) Standards and guidelines for the interpretation of sequence variants: a joint consensus recommendation of the American College of Medical Genetics and Genomics and the Association for Molecular Pathology. Genet Med 17:405-424. https://doi.org/10.1038/gim.2015.30

Rinaldi F, Clematide S, Marques H, Ellendorff T, Romacker M, Rodriguez-Esteban R (2014) OntoGene web services for biomedical text mining. BMC Bioinform 15(Suppl 14):S6. https://doi. org/10.1186/1471-2105-15-S14-S6

Rink B, Harabagiu S, Roberts K (2011) Automatic extraction of relations between medical concepts in clinical texts. J Am Med Inform Assoc 18:594-600. https://doi.org/10.1136/amiajnl-2011000153

Rocktaschel T, Weidlich M, Leser U (2012) ChemSpot: a hybrid system for chemical named entity recognition. Bioinformatics 28:1633-1640. https://doi.org/10.1093/bioinformatics/bts183

Sajda P (2006) Machine learning for detection and diagnosis of disease. Annu Rev Biomed Eng 8:537-565. https://doi.org/10.1146/annur ev.bioeng.8.061505.095802

Sanchez-Vega F, Mina M, Armenia J, Chatila WK, Luna A, La KC, Dimitriadoy S, Liu DL, Kantheti HS, Saghafinia S, Chakravarty D, Daian F, Gao Q, Bailey MH, Liang WW, Foltz SM, Shmulevich I, Ding L, Heins Z, Ochoa A, Gross B, Gao J, Zhang H, Kundra R, Kandoth C, Bahceci I, Dervishi L, Dogrusoz U, Zhou W, Shen H, Laird PW, Way GP, Greene CS, Liang H, Xiao Y, Wang C, Iavarone A, Berger AH, Bivona TG, Lazar AJ, Hammer GD, Giordano T, Kwong LN, McArthur G, Huang C, Tward AD, Frederick MJ, McCormick F, Meyerson M, Cancer Genome Atlas Research Network, Van Allen EM, Cherniack AD, Ciriello G, Sander C, Schultz N (2018) Oncogenic signaling pathways in the cancer genome atlas. Cell 173(2):321-337.e10. https://doi.org/10.1016/j. cell.2018.03.035

Santana LS et al (2017) Clinical application of ACMG-AMP guidelines in HNF1A and GCK variants in a cohort of MODY families. Clin Genet 92:388-396. https://doi.org/10.1111/cge.12988

Settles B (2005) ABNER: an open source tool for automatically tagging genes, proteins and other entity names in text. Bioinformatics 21:3191-3192. https://doi.org/10.1093/bioinformatics/bti475

Singhal A, Simmons M, Lu Z (2016) Text mining for precision medicine: automating disease-mutation relationship extraction from biomedical literature. J Am Med Inform Assoc 23:766-772. https ://doi.org/10.1093/jamia/ocw041

Song M, Kim WC, Lee D, Heo GE, Kang KY (2015) PKDE4J: entity and relation extraction for public knowledge discovery. J Biomed Inform 57:320-332. https://doi.org/10.1016/j.jbi.2015.08.008

Spinella JF et al (2016) SNooPer: a machine learning-based method for somatic variant identification from low-pass next-generation sequencing. BMC Genom 17:912. https://doi.org/10.1186/s1286 4-016-3281-2

Stajkovska A, Mehandziska S, Stavrevska M, Jakovleva K, Nikchevska N, Mitrev Z, Kungulovski I, Zafiroski G, Tasic V, Kungulovski G (2018) Trio clinical exome sequencing in a patient with multicentric carpotarsal osteolysis syndrome: first case report in the Balkans. Front Gene 9:113. https://doi.org/10.3389/fgene.2018.00113

Steuer CE, Ramalingam SS (2018) Tumor mutation burden: leading immunotherapy to the era of precision medicine? J Clin Oncol 36:631-632. https://doi.org/10.1200/JCO.2017.76.8770 
Sukhai MA et al (2016) A classification system for clinical relevance of somatic variants identified in molecular profiling of cancer. Genet Med 18:128-136. https://doi.org/10.1038/gim.2015.47

Syrjala KL (2018) Opportunities for improving oncology care. Lancet Oncol 19:449. https://doi.org/10.1016/S1470-2045(18)30208-0

Szymczak S, Biernacka JM, Cordell HJ, Gonzalez-Recio O, Konig IR, Zhang H, Sun YV (2009) Machine learning in genome-wide association studies. Genet Epidemiol 33(Suppl 1):S51-S57. https ://doi.org/10.1002/gepi.20473

Tang H, Thomas PD (2016) Tools for predicting the functional impact of nonsynonymous genetic variation. Genetics 203:635-647. https://doi.org/10.1534/genetics.116.190033

Telenti A, Lippert C, Chang PC, DePristo M (2018) Deep learning of genomic variation and regulatory network data. Hum Mol Genet 27:R63-R71. https://doi.org/10.1093/hmg/ddy115

The Health Insurance Portability and Accountability Act of 1996 (2014) Pub. L. 104-191. Stat (1936) Web. 11 Aug. 2014. https:// www.gpo.gov/fdsys/pkg/PLAW-104publ191/content-detail.html. Accessed 10 Sept 2018

Thomas P, Neves M, Solt I, Tikk D, Leser U (2011) relation extraction for drug-drug interactions using ensemble learning. In: Proceedings of the 1st challenge task on drug-drug interaction extraction (DDIExtraction 2011), Huelva, pp 11-18

Thomas P, Rocktaschel T, Hakenberg J, Lichtblau Y, Leser U (2016) SETH detects and normalizes genetic variants in text. Bioinformatics 32:2883-2885. https://doi.org/10.1093/bioinformatics/ btw234

Thompson BA et al (2014) Application of a 5-tiered scheme for standardized classification of 2,360 unique mismatch repair gene variants in the InSiGHT locus-specific database. Nat Genet 46:107-115. https://doi.org/10.1038/ng.2854

Tikk D, Thomas P, Palaga P, Hakenberg J, Leser U (2010) A comprehensive benchmark of kernel methods to extract protein-protein interactions from literature. PLoS Comput Biol 6:e1000837. https://doi.org/10.1371/journal.pcbi.1000837

Tikk D, Solt I, Thomas P, Leser U (2013) A detailed error analysis of 13 kernel methods for protein-protein interaction extraction. BMC Bioinform 14:12. https://doi.org/10.1186/1471-2105-14-12

Tsuruoka Y, Miwa M, Hamamoto K, Tsujii J, Ananiadou S (2011) Discovering and visualizing indirect associations between biomedical concepts. Bioinformatics 27:i111-i119. https://doi. org/10.1093/bioinformatics/btr214

Vaser R, Adusumalli S, Leng SN, Sikic M, Ng PC (2016) SIFT missense predictions for genomes. Nat Protoc 11:1-9. https://doi. org/10.1038/nprot.2015.123

Vaske CJ, Benz SC, Sanborn JZ, Earl D, Szeto C, Zhu J, Haussler D, Stuart JM (2010) Inference of patient-specific pathway activities from multi-dimensional cancer genomics data using PARADIGM. Bioinformatics 26(12):i237-i245. https://doi. org/10.1093/bioinformatics/btq182

Wainberg M, Merico D, Delong A, Frey BJ (2018) Deep learning in biomedicine. Nat Biotechnol 36(9):829-838. https://doi. org/10.1038/nbt.4233

Wainreb G et al (2010) MuD: an interactive web server for the prediction of non-neutral substitutions using protein structural data. Nucleic Acids Res 38:W523-W528. https://doi.org/10.1093/nar/ gkq528

Wang B, Mezlini AM, Demir F, Fiume M, Tu Z, Brudno M, HaibeKains B, Goldenberg A (2014) Similarity network fusion for aggregating data types on a genomic scale. Nat Methods. 11(3):333-7. https://doi.org/10.1038/nmeth.2810

Wartman SA, Combs CD (2018) Medical education must move from the information age to the age of artificial intelligence. Acad Med 93(8):1107-1109. https://doi.org/10.1097/ACM.00000 00000002044

Wei CH, Harris BR, Kao HY, Lu Z (2013) tmVar: a text mining approach for extracting sequence variants in biomedical literature. Bioinformatics 29:1433-1439. https://doi.org/10.1093/bioin formatics/btt156

Wei CH, Kao HY, Lu Z (2015) GNormPlus: an integrative approach for tagging genes, gene families, and protein domains. Biomed Res Int 2015:918710. https://doi.org/10.1155/2015/918710

Wood DE et al (2018) A machine learning approach for somatic mutation discovery. Sci Transl Med. https://doi.org/10.1126/scitr anslmed.aar7939

Wu Y, Jiang M, Xu J, Zhi D, Xu H (2017) Clinical named entity recognition using deep learning models. AMIA Annu Symp Proc 2017:1812-1819

Xu R, Wang Q (2013) A semi-supervised approach to extract pharmacogenomics-specific drug-gene pairs from biomedical literature for personalized medicine. J Biomed Inform 46:585-593. https ://doi.org/10.1016/j.jbi.2013.04.001

Xu Y, Hong K, Tsujii J, Chang EI (2012) Feature engineering combined with machine learning and rule-based methods for structured information extraction from narrative clinical discharge summaries. J Am Med Inform Assoc 19:824-832. https://doi. org/10.1136/amiajnl-2011-000776

Yang Z, Tang N, Zhang X, Lin H, Li Y, Yang Z (2011) Multiple kernel learning in protein-protein interaction extraction from biomedical literature. Artif Intell Med 51:163-173. https://doi.org/10.1016/j. artmed.2010.12.002

Yu P et al (2017) FGF-dependent metabolic control of vascular development. Nature 545:224-228. https://doi.org/10.1038/natur e22322

Yuan Y, Shi Y, Li C, Kim J, Cai W, Han Z, Feng DD (2016) DeepGene: an advanced cancer type classifier based on deep learning and somatic point mutations. BMC Bioinform 17:476. https://doi. org/10.1186/s12859-016-1334-9

Yue P, Moult J (2006) Identification and analysis of deleterious human SNPs. J Mol Biol 356:1263-1274. https://doi.org/10.1016/j. jmb.2005.12.025

Zhang T, Yan L, Leng Y, Chen C, Ma L, Wang Q, Zhang J, Cao L (2018) A novel missense mutation of CRYGS underlies congenital cataract in a Chinese family. Gene 675:9-14. https://doi. org/10.1016/j.gene.2018.06.100

Zomnir MG, Lipkin L, Pacula M, Meneses ED, MacLeay A, Duraisamy S, Nadhamuni N, Al Turki SH, Zheng Z, Rivera M, Nardi V, Dias-Santagata D, Iafrate AJ, Le LP, Lennerz JK (2018) Artificial intelligence approach for variant reporting. JCO Clin Cancer Inform. https://doi.org/10.1200/CCI.16.00079

Zou Q, Lamurias A, Clarke LA, Couto FM (2017) Extracting microRNA-gene relations from biomedical literature using distant supervision. Plos One. https://doi.org/10.1371/journ al.pone.0171929

Publisher's Note Springer Nature remains neutral with regard to jurisdictional claims in published maps and institutional affiliations. 